\section{Hepatitis sifilítica: reporte de una serie de casos}

\author{
GABRIEL MEZZANO ${ }^{1,2}$, FRANCISCO RONDÓN ${ }^{1, a}$, \\ ALLYSON CID $^{1, b}$, FELIPE PARRA ${ }^{1, b}$, ANDRÉS SOTO $^{3,4}$, \\ FERNANDO GÓMEZ ${ }^{1}$, DANIELA JARA ${ }^{1}$, MARÍA ISABEL JIRÓN ${ }^{1,4}$
}

\section{Syphilitic hepatitis. Report of three cases}

Liver involvement occurs in 0.2 to 3\% of patients with syphilis. We report three patients with liver involvement in syphilis. A 52-year-old male presenting with erythema and malaise. Laboratory showed a gamma glutamyl transpeptidase (GGT) of $853 \mathrm{u} / \mathrm{l}$, alkaline phosphatases of 1,010 U/L and VDRL was positive. Treatment with penicillin resolved the skin problem and normalized liver enzymes. A HIV positive 30-year-old male in peritoneal dialysis presenting with itching, malaise and markedly elevated GGT and alkaline phosphatases. VDRL was positive. He was treated with penicillin with remission of symptoms and enzyme normalization. A 43-year-old male presenting with erythema, malaise, arthralgias and elevated GGT and alkaline phosphatases. VDRL was positive and treatment with penicillin reverted symptoms and laboratory abnormalities.

(Rev Med Chile 2019; 147: 251-255)

Key words: Hepatitis; Sexually Transmitted Diseases; Syphilis.
'Sección de Gastroenterología, Hospital del Salvador. Santiago, Chile.

${ }^{2}$ Servicio Gastroenterología, Clínica Universidad de los Andes. Santiago, Chile.

${ }^{3}$ Sección de Infectología, Hospital del Salvador. Santiago, Chile.

${ }^{4}$ Facultad de Medicina,

Universidad de Chile. Santiago,

Chile.

aResidente Gastroenterología.

${ }^{\mathrm{b}}$ Residente Medicina Interna.

Recibido el 17 de agosto de 2018, aceptado el 22 de enero de 2019.

Correspondencia a: Gabriel Mezzano Sección Gastroenterología, Servicio Medicina Interna, Hospital del Salvador, Avenida Salvador 364. Santiago, Chile. Gmezzano@hsalvador.cl
E n los últimos 3 años existe un aumento significativo en la incidencia de todas las infecciones de transmisión sexual (ITS) en Chile, incluyendo sífilis, infección por el virus de la inmunodeficiencia humana (VIH), hepatitis B, gonorrea ${ }^{1}$, e incluso brotes de hepatitis A por mecanismo de transmisión fecal oral en hombres que tienen sexo con hombres (HSH), lo que refleja un problema nacional, un fracaso en las medidas de salud pública para su diagnóstico y manejo oportuno, y una deficiencia de origen multifactorial en la aplicación de medidas óptimas de prevención.

La sífilis es una enfermedad infecciosa producida por el Treponema pallidum, donde la mayoría de los contagios son secundarios a transmisión sexual y pueden manifestarse clínicamente como sífilis primaria, secundaria, terciaria o latente ${ }^{2}$. La incidencia de sífilis en Estados Unidos de Norteamérica ha presentado un aumento sostenido en los últimos años ${ }^{3}$, así como en Chile ${ }^{1}$, en particular la prevalencia de coinfección sífilis y VIH se describe en torno a $21 \%{ }^{4}$.
La afectación hepática es un compromiso poco habitual de la sífilis primaria y secundaria, descrita en aproximadamente $0,2-3 \%$, siendo más frecuente en pacientes $\mathrm{VIH}$, ya que comparten factores de riesgo similares ${ }^{5,6}$.

El diagnóstico diferencial se plantea en pacientes con alteración del perfil hepático, principalmente de carácter colestásico, en los que se han descartado las causas más frecuentes mediante técnicas de laboratorio e imagenológicas ${ }^{7}$, asociado a un estudio serológico o molecular para Treponema pallidum.

Presentar esta serie de casos tiene como objetivo principal comunicar una manifestación poco frecuente y que plantea un importante desafío diagnóstico.

\section{Casos clínicos}

Caso 1

Paciente masculino de 51 años. Presentaba cuadro de 3 semanas caracterizado por lesiones 
eritematosas en cara, tronco y extremidades, asociado a sensación febril, compromiso general y orina espumosa, además, edema escrotal y de extremidades inferiores. Al examen físico se observaba exantema maculopapular generalizado que comprometía plantas y edema de extremidades inferiores (Figura 1). En el estudio de laboratorio destacaba: en el hemograma, leucocitos 10.100 $\mathrm{U} / \mathrm{ml}$, plaquetas $438.000 \mathrm{U} / \mathrm{ml}$, INR 1,0, PCR 35 $\mathrm{mg} / \mathrm{dl}$, nitrógeno ureico $29 \mathrm{mg} / \mathrm{dl}$, creatinina 1,02 $\mathrm{mg} / \mathrm{dl}$, orina completa: leucocitos 4-8 x campo, proteinuria aislada $500 \mathrm{mg} / \mathrm{dl}$; albuminemia 2,2 $\mathrm{g} / \mathrm{dl}$; bilirrubinemia total/directa $1,5 / 0,23 \mathrm{mg} /$ $\mathrm{dl}$, aspartato aminotransferasa (AST) $78 \mathrm{U} / \mathrm{L}$, alanina aminotransferasa (ALT) $73 \mathrm{U} / \mathrm{L}$, fosfatasas alcalinas (FA) $1.010 \mathrm{U} / \mathrm{L}$, gamma glutamil transpeptidasa (GGT) 853 U/L. Serología para VIH, HBsAg, anticore total virus B y anticuerpos hepatitis $\mathrm{C}$ no reactivas.

Se planteó el diagnóstico de síndrome nefrótico y síndrome colestásico en estudio. Se complementó el estudio con anticuerpos antinucleares (AAN) (-), ANCA (+) patrón citoplasmático, anticuerpos antimitocondriales (AAM) (-), electroforesis de proteínas con hipoalbuminemia e hipergammaglobulinemia difusa, VHS 123 $\mathrm{mm} / \mathrm{h}$, proteinuria $24 \mathrm{~h} 560 \mathrm{mg} / 2.500 \mathrm{ml}, \mathrm{C} 3 \mathrm{y}$ C4 normales, cuantificación sérica de IgG e IgA normales, IgM 2,5 veces sobre el valor normal, perfil tiroideo normal. VDRL reactivo en dilución de 1:64. Tomografía computada de abdomen y pelvis dentro de límites normales. Biopsia renal compatible con glomerulonefritis membranosa por complejos inmunes IgA, IgG e IgM.

Se planteó cuadro de sífilis secundaria con compromiso hepático y renal. VDRL en líquido céfalo-raquídeo (LCR) fue negativo. Se indicó tratamiento con penicilina benzatina 2,4 millones UI IM semanal por 2 semanas. Evolucionó favorablemente, con desaparición de exantema, mejoría de hipoalbuminemia y resolución del patrón colestásico.

\section{Caso 2}

Paciente masculino de 30 años de edad, con antecedente de enfermedad renal crónica en diálisis peritoneal secundaria a hidronefrosis congénita, coinfección VIH y hepatitis $B$, en terapia antirretroviral, ambos virus con carga viral indetectable y recuento de linfocitos CD4 196 células/ $\mathrm{ml}$. Consultó por 1 mes de prurito generalizado y compromiso del estado general. Al examen físico destacaba hepatomegalia y esplenomegalia y perfil hepático con patrón colestásico, FA 1.092 U/L, GGT $958 \mathrm{U} / \mathrm{L}$, con bilirrubina total/directa $0,2 / 0,1$ $\mathrm{mg} / \mathrm{dl}$, AST $40 \mathrm{U} / \mathrm{L}$ y ALT $52 \mathrm{U} / \mathrm{L}$.

Estudio de AAN, AAM, AML y recuento de inmunoglobulinas normales, IgM VHA y anticuerpos anti-VHC no reactivos. Ecografía abdominal con signos de hepatitis aguda, sin alteraciones de vía biliar y colangiorresonancia sin alteraciones.

Se inició empíricamente ácido ursodeoxicólico $1.000 \mathrm{mg}$ al día con buena respuesta clínica y de laboratorio al mes de tratamiento, con disminución significativa de fosfatasas alcalinas (Tabla 1). Se complementó el estudio con VDRL en sangre, que resultó reactivo con títulos de 1:128 y punción lumbar, que descartó neurolúes.

Se indicó penicilina benzatina 2,4 millones UI intramuscular en una dosis semanal, por dos semanas y suspensión del ácido ursodeoxicólico, con lo que logró remisión completa de la sintomatología y normalización del perfil hepático en 5 semanas.

\section{Caso 3}

Paciente masculino de 43 años, sin antecedentes mórbidos, con historia de 3 semanas de compromiso del estado general, fiebre intermi-

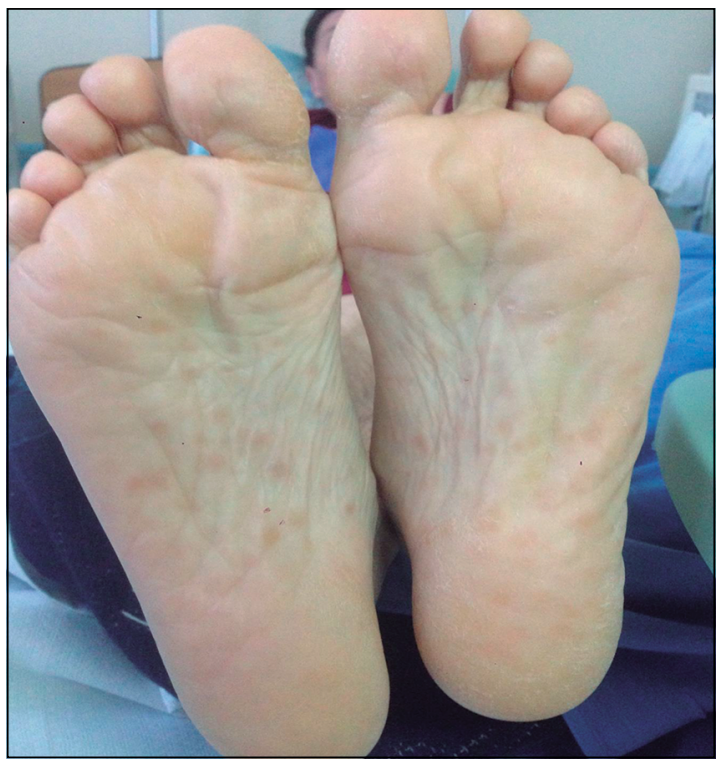

Figura 1. Exantema maculopapular generalizado que compromete plantas. 
Tabla $1^{16}$

\begin{tabular}{|c|c|c|c|c|c|c|c|c|c|c|c|c|}
\hline & Sexo & Edad & VIH & $\begin{array}{c}\text { Sífilis } \\
\text { previa }\end{array}$ & VDRL & Estadío & ALT & AST & FA & GGT & $\begin{array}{l}\text { Patologías } \\
\text { descartadas }\end{array}$ & Tratamiento \\
\hline 1 & M & 30 & Sí & No & $1 / 128$ & $\begin{array}{c}\text { Sífilis } \\
\text { secundaria }\end{array}$ & 40 & 54 & 1.092 & 958 & $\begin{array}{l}\text { Serología para ANA, VHA } \\
\text { y VHC negativas. Sin his- } \\
\text { toria de abuso de alcohol } \\
\text { ni uso de fármacos. } \\
\text { Inmunoglobulinas G, A y } \\
\text { M recuento normal. AML } \\
\text { negativo. VHB tratado, } \\
\text { carga viral negativa }\end{array}$ & $\begin{array}{l}\text { Penicilina benza- } \\
\text { tina } 2.400 .000 \\
\text { semanal, } \\
2 \text { dosis }\end{array}$ \\
\hline 2 & M & 51 & No & No & $1 / 64$ & $\begin{array}{c}\text { Sífilis } \\
\text { secundaria }\end{array}$ & 78,6 & 73,4 & 1.010 & 853 & $\begin{array}{l}\text { Serología para ANA, VHB } \\
\text { y VHC. Sin historia de } \\
\text { abuso de alcohol ni uso } \\
\text { de fármacos. Inmuno- } \\
\text { globulinas G, A y M } \\
\text { recuento normal }\end{array}$ & $\begin{array}{c}\text { Penicilina benza- } \\
\text { tina } 2.400 .000 \\
\text { semanal, } \\
2 \text { dosis }\end{array}$ \\
\hline 3 & M & 43 & No & No & $1 / 16$ & $\begin{array}{c}\text { Sífilis } \\
\text { secundaria }\end{array}$ & 136 & 281 & 298 & 314 & $\begin{array}{l}\text { Serología para VHA, VHB } \\
\text { y VHC. Sin historia de } \\
\text { abuso de alcohol ni uso } \\
\text { de fármacos }\end{array}$ & $\begin{array}{c}\text { Penicilina benza- } \\
\text { tina } 2.400 .000 \\
\text { semanal, } \\
2 \text { dosis }\end{array}$ \\
\hline
\end{tabular}

tente y exantema no pruriginoso de predominio en tórax. A la semana de inicio de síntomas se agregó artralgias de codos, rodillas y muñecas, sin aumento de volumen en articulaciones ni rigidez matinal. En exámenes de laboratorio destacaba GGT 314 U/L, FA 298 U/L, AST 136 U/L, ALT 281 $\mathrm{U} / \mathrm{L}$, bilirrubina total/directa $0,6 / 0,3 \mathrm{mg} / \mathrm{dl}, \mathrm{LDH}$ $205 \mathrm{U} / \mathrm{L}$ y creatinina $0,76 \mathrm{mg} / \mathrm{dl}$. Por la presencia de exantema y colestasia se solicitó VDRL que resultó positivo 1/16, VIH no reactivo, HBsAg negativo, anti-VHC negativo, IgM VHA negativo. Ecografía abdominal con leve hepatomegalia, vía biliar normal, sin otros hallazgos.

Se indicó tratamiento con penicilina benzatina 2,4 millones UI im/semanal por dos veces. El paciente presentó mejoría clínica y normalización de las pruebas hepáticas a las 4 semanas: bilirrubina total/directa $0,5 / 0,2 \mathrm{mg} / \mathrm{dl}$, GGT $28 \mathrm{U} / \mathrm{L}, \mathrm{FA} 83$ U/L, AST 24 U/L, ALT 23 U/L.

\section{Discusión}

El aumento de las infecciones de transmisión sexual en los últimos años plantea el desafío de optimizar su diagnóstico y prevención. La hepatitis sifilítica es una entidad clínica poco frecuente, que ha sido descrita en solo 50 casos en la literatura desde el año 1960, y de estos, mayoritariamente son pacientes con infección por $\mathrm{VIH}^{8}$.

La sífilis es un importante problema de salud pública a nivel mundial, especialmente en $\mathrm{HSH}$, pacientes con infección VIH, usuarios de drogas ilícitas y otros grupos de riesgo. Los datos nacionales de sífilis muestran una tasa de incidencia de 21,5 por 100.000 habitantes durante 2017 , siendo este número superior en $21 \%$ a lo registrado en mismo período del año 2016. Al igual que para el resto de las ETS, el grupo etario de mayor riesgo se concentra entre los 20 y 39 años ${ }^{1}$.

La sífilis es capaz de comprometer prácticamente cualquier órgano del cuerpo humano, siendo la hepatitis sifilítica una manifestación poco frecuente, descrita entre 0,7 y $2,7 \%$ en distintas series $^{5,9}$. El compromiso hepático se puede presentar en sífilis primaria, secundaria o terciaria, siendo la diseminación hematógena la principal hipótesis, ya que sugiere la migración del treponema a través del sistema venoso hasta la circulación hepática, esto planteado al demostrar la presencia del agente en las biopsias hepáticas y su desaparición posterior al tratamiento ${ }^{10-12}$.

Pese a los anterior, el diagnóstico se debe plantear en pacientes con factores de riesgo que 
presentan alteración del perfil hepático con un patrón principalmente colestásico, siendo descartadas las causas más frecuentes de esta, tanto para pacientes inmunocompetentes (coledocolitiasis, esteatohepatitis no alcohólica, medicamentos, hierbas, colangitis biliar primaria, colangitis esclerosante primaria) como para inmunosuprimidos

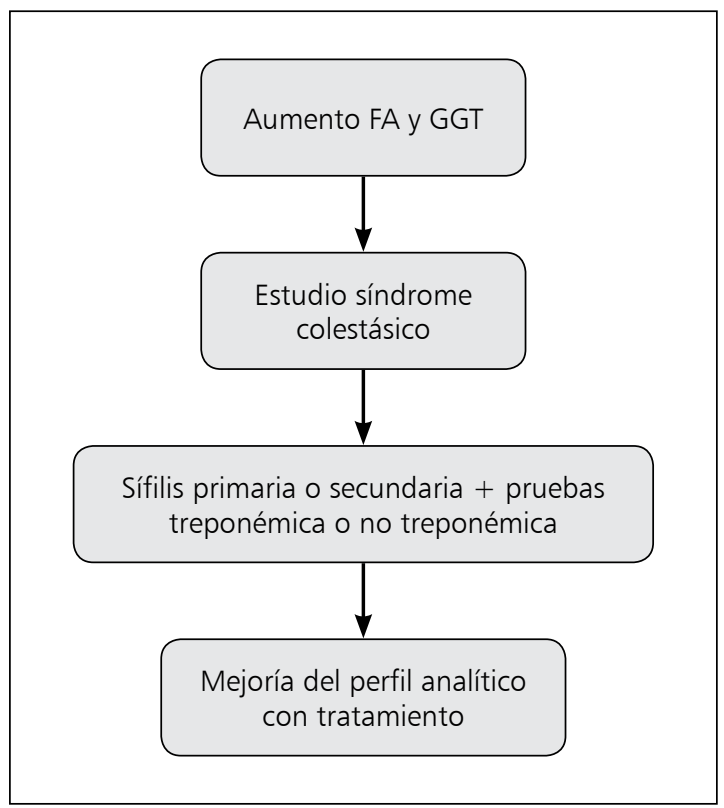

Figura 2. (citomegalovirus, micobacterias, criptosporidiasis, neoplasias como sarcoma de Kaposi o linfomas). A lo anterior se debe agregar la positividad en los tests serológicos no treponémicos (VDRL o RPR), treponémicos (FTA- ABS, MHA-TP, ELISA) o moleculares, independiente del título o positividad de estos. Se han planteado criterios diagnósticos que concentran lo expuesto previamente pero que no poseen cohortes de validación y están basados en series de pacientes analizados de manera retrospectiva (Figura 2) ${ }^{13}$.

El compromiso hepático por sífilis a nivel histológico es inespecífico, lo descrito es la presencia de un infiltrado linfocítico periportal con presencia variable de necrosis focal perivena central, espacio portal y lobulillar, con presencia ocasional de espiroquetas (Figura 3). En esta serie ningún paciente contó con biopsia hepática, ya que la elevada sospecha clínica y el estudio realizado permitieron una adecuada aproximación diagnóstica.

El tratamiento se basa en el estadio de la enfermedad y en la presencia de compromiso del sistema nervioso central. El Treponema pallidum aún presenta extrema susceptibilidad a la penicilina, tanto así que no existen reportes de sífilis resisten$t^{14}$. La recomendación actual para el tratamiento de la sífilis temprana (primaria y secundaria) es la administración de una dosis semanal de penicilina $\mathrm{G}$ benzatina 2,4 millones UI, dos semanas seguidas ${ }^{15}$, con lo que se reportan tasas de curación

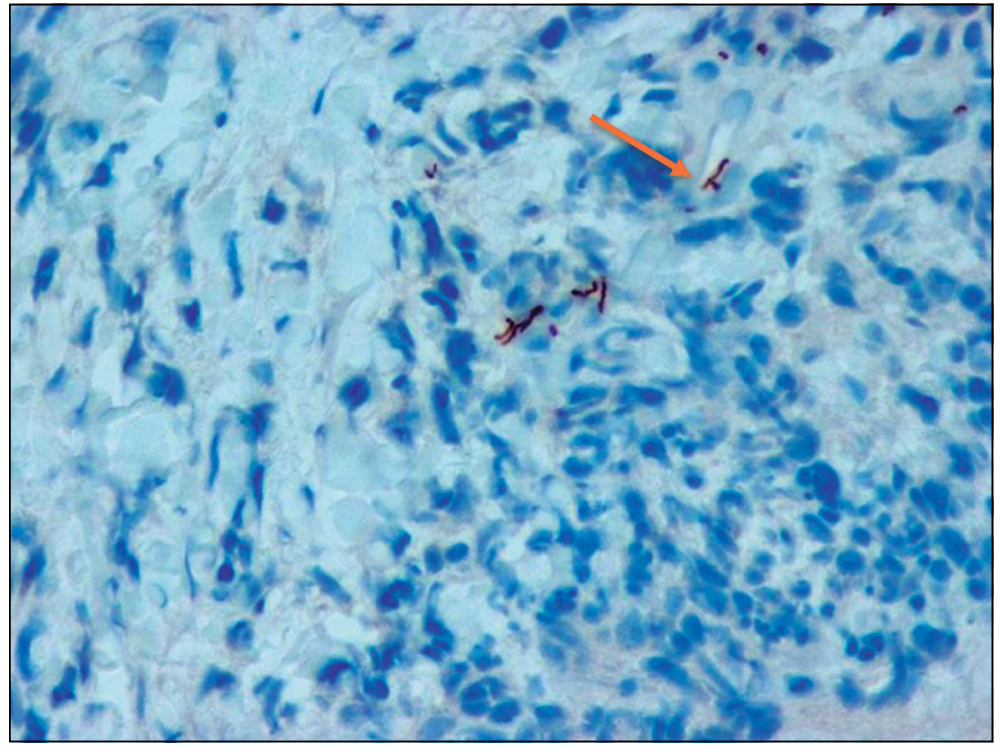

Figura 3. Adaptado de Rubio-Tapia et al. Hepatology 2013; 1-23. Presencia de espiroquetas en biopsia hepática. 
entre $80-95 \%$ a los 6 meses de seguimiento. Otras alternativas al tratamiento estándar es el uso de doxiciclina, ceftriaxona, tetraciclina o azitromicina (evidencia grado B) ${ }^{2}$. Los casos reportados de hepatitis sifilítica fueron tratados con PNC G benzatina (con 1 a 3 dosis), presentando normalización del perfil hepático entre las 4 y 16 semanas posteriores $^{11,16}$. Por lo antes descrito, es importante incorporar la solicitud de pruebas treponémicas y no treponémicas en aquellos pacientes que se presentan con alteración del perfil hepático de tipo colestásico en quienes el estudio habitual de colestasia no permite esclarecer el diagnóstico. De nuestro conocimiento, no existe otra serie de casos sobre hepatitis sifilítica en Chile.

\section{Referencias}

1. Ministerio de Salud Chile. Boletín Epidemiológico Trimestral, Sífilis en todas sus formas. Vol. 113, Minsal. 2017.

2. Clement ME, Okeke NL, Hicks CB. Treatment of Syphilis. JAMA 2014; 312 (18): 1905.

3. Rubio-Tapia A, Hujoel IA, Smyrk TC, Poterucha JJ. Emerging secondary syphilis presenting as syphilitic hepatitis. Hepatology 2017; 65 (6): 2113-5.

4. Pérez CC, Cerón AI, Fuentes LG, Zañartu SC, Balcells MME, Ajenjo HC, et al. Coinfecciones por virus hepatitis B, virus hepatitis C, Treponema pallidum y Toxoplasma gondii en la cohorte de pacientes VIH positivos en control en la Pontificia Universidad Católica de Chile. Rev Med Chile 2009; 137 (5): 641-8.

5. García A, Paredes G De, Villajos LT, Castro LA, de Santiago ER, Aracil CF, et al. Hepatitis aguda como única manifestación de sífilis en un paciente inmunocompetente. Gastroenterol Latinoam 2015; 45 (3): 230-2.

6. Tolia S, Kassem H, Capatina-Rata A. The Great Impostor: Transaminitis Masking the Coinfection of Syphilis and Human Immunodeficiency Virus. Case Rep Med 2017 (DOI: 10:1155/2017/2481961).

7. Association E. EASL Clinical Practice Guidelines: Management of cholestatic liver diseases. J Hepatol 2009; 51 (2): 237-67.

8. Mandache C, Coca C, Caro-Sampara F, Haberstezer F, Coumaros D, Blicklé F, et al. A forgotten aetiology of acute hepatitis in immunocompetent patient: Syphilitic infection [1]. J Intern Med 2006; 259 (2): 214-5.

9. Aggarwal SK, Radhakrishnan S. Syphilitic hepatitis: Look for raised alkaline phosphatase level. Med J Armed Forces India 2016; 72 (2): 192-3.

10. Adachi E, Koibuchi T, Okame M, Sato H, Imai K, Shimizu $\mathrm{S}$, et al. Case of secondary syphilis presenting with unusual complications: Syphilitic proctitis, gastritis, and hepatitis. J Clin Microbiol 2011; 49 (12): 4394-6.

11. Kim GH, Kim BU, Lee JH, Choi YH, Chae HB, Park $\mathrm{SM}$, et al. Cholestatic hepatitis and thrombocytosis in a secondary syphilis patient. J Korean Med Sci 2010; 25 (11): 1661-4.

12. Fehér J, Somogyi T, Timmer M, Józsa L. Early syphilitic hepatitis. Lancet (London, England. 1975; 2 (7941): 8969.

13. Mullick C, Liappis A, Benator D, Roberts A, Parenti D, Simon G. Syphilitic hepatitis in HIV-infected patients: a report of 7 cases and review of the literature. Clin Infect Dis 2004; 39 (10): e100-5.

14. Lewis DA, Lukehart SA. Antimicrobial resistance in Neisseria gonorrhoeae and Treponema pallidum: Evolution, therapeutic challenges and the need to strengthen global surveillance. Sex Transm Infect 2011; 87 (SUPPL. 2): 39-43.

15. Ministerio de Salud de Chile. Norma de Profilaxis, Diagnóstico y Tratamiento de las Infecciones de Transmisión Sexual (ITS). ISBN: 978-956-348-103-7 (Disponible en: www.minsal.cl).

16. Murphy CJ, Bhatt A, Chen W, Malli A, Mcgorisk T, Kelly SG. Clinical Picture Syphilitic hepatitis. Lancet Gastroenterol Hepatol 2017; 2 (12): 920. 